\title{
Triangulability of Convex Graphs and Convex Skewness
}

\author{
Niran Abbas $\mathrm{Ali}^{a}$, Gek L. Chia ${ }^{b, e}$, Hazim Michman Trao ${ }^{c}$ and Adem Kilicman ${ }^{d}$ \\ ${ }^{a, c, d}$ Department of Mathematics, \\ Universiti Putra Malaysia, 43400 Serdang, Malaysia, \\ ${ }^{b}$ Department of Mathematical and Actuarial Sciences, \\ Universiti Tunku Abdul Rahman, Sungai Long Campus, Malaysia \\ ${ }^{e}$ Institute of Mathematical Sciences, University of Malaya, \\ 50603 Kuala Lumpur, Malaysia
}

\begin{abstract}
Motivated by a result of [1] which states that if $F$ is a subgraph of a convex complete graph $K_{n}$ and $F$ contains no boundary edge of $K_{n}$ and $|E(F)| \leq n-3$, then $K_{n}-F$ admits a triangulation, we determine necessary and sufficient conditions on $F$ with $|E(F)| \leq n-1$ for which the conclusion remains true. For $|E(F)| \geq n$, we investigate the possibility of packing $F$ in $K_{n}$ such that $K_{n}-F$ admits a triangulation for certain families of graphs $F$. These results are then applied to determine the convex skewness of the convex graphs of the form $K_{n}-F$.
\end{abstract}

\section{Introduction and Preliminary}

By a geometric graph we mean a graph whose edges are straight line segments. By a convex graph, we mean a geometric graph whose vertices are in convex position. Let $G$ be a geometric graph with $n$ vertices having $k$ vertices in the convex hull. By a triangulation of $G$ we mean a maximal planar subdivision with the vertex set $V(G)$ of $G$. Hence a triangulation of $G$ has $2 n-2-k$ triangles and $3 n-3-k$ edges. It has been shown in [8] that the problem of deciding whether a given geometric graph admits a triangulation of its vertex set is an $N P$-complete problem.

For the case where $G$ is a convex geometric graph, it has been proved in [1] that $G$ admits a triangulation if $G$ is obtained from the complete convex graph $K_{n}$ by deleting a set $F$ of at most $n-3$ edges and $F$ contains no edges from the boundary of the convex hull of $G$ (see Theorem 11). It was noted that the result is best possible with respect to the number of edges in $G$. Motivated by this, we wish to obtain conditions on $F$ for which the result remains true when $F$ has more than $n-3$ edges.

Throughout, if $F$ is subgraph of $K_{n}$, then $K_{n}-F$ will denote the convex graph obtained from $K_{n}$ by deleting the set of edges of $F$.

Theorem 1 ([1] (Theorem 4.1))

Let $F$ be a subgraph of a convex complete graph $K_{n}$. Suppose $F$ contains no boundary edge of $K_{n}$ and $|E(F)| \leq n-3$. Then $K_{n}-F$ admits a triangulation. 
For $F$ having at most $n-1$ edges, we obtain necessary and sufficient conditions on $F$ such that $K_{n}-F$ admits a triangulation. These conditions are given in Propositions 1 and 2, and Theorems 2 and 3 .

The case where $F$ has at least $n$ edges seems to be a little complicated. For this we turn to look for a configuration for $F$ that can be packed in $K_{n}$ so that $K_{n}-F$ admits a triangulation. If such a configuration exists for $F$, then we say that $F$ is potentially triangulable in $K_{n}$. Potentially triangulable graphs are considered in Section 4 where we (i) determine precisely the value of $n$ for which the $n$-cycle is potentially triangulable in $K_{n}$ (Theorem 44), and (ii) characterize all 2-regular graphs which are potentially triangulable in $K_{n}$ (Theorem 5).

The potentially triangulable problem is extended to the regular case in Section 5. Here, while unable to solve the general case, we consider the generalized Petersen graph.

We end the paper by showing an application of these result to the problem of determining the minimum number of edges to be deleted from a given convex graph so that the resulting graph is a convex plane graph.

Throughout, we shall adopt the following notations. Unless otherwise stated, the vertices of a convex complete graph $K_{n}$ will be denoted by $v_{0}, v_{1}, \ldots, v_{n-1}$ in cyclic ordered. Also, unless otherwise specified, any operation on the subscript of $v_{i}$ is reduced modulo $n$.

Lemma 1 Let $F$ be a subgraph of a convex complete graph $K_{n}$. Assume that $F$ has at most $n-1$ edges and having no boundary edge of $K_{n}$. Then $K_{n}-F$ has a vertex $v_{i}$ such that $v_{i-1} v_{i+1}$ is not an edge of $F$.

Proof: If the lemma is not true, then it implies that $v_{i-1} v_{i+1}, v_{i-2} v_{i}, v_{i} v_{i+2} \in E(F)$, and recursively, this implies that $F$ is a spanning subgraph of $K_{n}$ with minimum vertexdegree at least 2. But this implies that $|E(F)| \geq n$, a contradiction.

\section{Graphs with at most $n-2$ edges}

We begin by describing a configuration for $F_{n}$ with $n$ vertices and $n-2$ edges such that $K_{n}-F$ admits no triangulation.

Definition 1 Let $F_{n}$ denote a subgraph of $K_{n}$ with no isolated vertices and having $n-2$ edges. Let $\mathcal{F}_{n}\left({ }^{*}\right)$ denote a configuration of $F_{n}$ on $K_{n}$ such that either $E\left(\mathcal{F}_{n}\left({ }^{*}\right)\right)=$ $\left\{v_{0} v_{i}, v_{1} v_{n-1} \mid i=2,3, \ldots, n-2\right\}$ or else $\mathcal{F}_{n}\left({ }^{*}\right)$ satisfies the following conditions:

(i) For $i=0,1,2, \ldots, k-1, d\left(v_{i}\right) \geq 2$ and $v_{i-1} v_{i+1} \in E\left(\mathcal{F}_{n}\left({ }^{*}\right)\right)$ where $k$ is some natural number with $2 \leq k \leq n-4$.

(ii) For $i=k, k+1, \ldots, n-1, d\left(v_{i}\right)=1$ and $v_{i}$ is adjacent to some non-pendant vertex in $\mathcal{F}_{n}\left({ }^{*}\right)$. 
(iii) For any two pendant vertices $u, v$ such that $u v_{i}$ and $v v_{j}$ are crossed in $\mathcal{F}_{n}\left({ }^{*}\right)$, then $|i-j|=1$.

Example 1 Let $F$ be a subgraph be a subgraph of $K_{10}$ with vertex set $\left\{v_{0}, v_{1}, \ldots, v_{9}\right\}$ and edge set $\left\{v_{0} v_{2}, v_{0} v_{8}, v_{1} v_{3}, v_{1} v_{7}, v_{1} v_{9}, v_{3} v_{5}, v_{3} v_{6}, v_{2} v_{4}\right\}$. Then $F$ is an example of a $\mathcal{F}_{10}\left({ }^{*}\right)$ configuration in $K_{10}$. Here $k=4$.

Note that in the above definition of $\mathcal{F}_{n}\left(^{*}\right)$, if $w \in\left\{v_{0}, v_{k-1}\right\}$, then $w$ is adjacent to some pendant vertex of $\mathcal{F}_{n}$.

Proposition 1 Suppose $F_{n}$ is a subgraph of the convex complete graph $K_{n}$ with a configuration $\mathcal{F}_{n}\left({ }^{*}\right)$ as defined above. Then $K_{n}-\mathcal{F}_{n}\left({ }^{*}\right)$ admits no triangulation for any natural number $n \geq 4$.

Proof: We prove this by induction on $n$. The result is clearly true if $4 \leq n \leq 6$. Hence we assume that $n \geq 7$ and that the result is true for all convex graphs $K_{m}-\mathcal{F}_{m}\left({ }^{*}\right)$ where $m<n$.

By Lemma 1, $K_{n}-\mathcal{F}_{n}\left({ }^{*}\right)$ contains a vertex $v_{i}$ such that $v_{i-1} v_{i+1} \notin E\left(\mathcal{F}_{n}\left({ }^{*}\right)\right)$. By the definition of $\mathcal{F}_{n}\left({ }^{*}\right)$, we see that $d_{\mathcal{F}_{n}\left({ }^{*}\right)}\left(v_{i}\right)=1$.

Let $F_{n-1}=\mathcal{F}_{n}\left({ }^{*}\right)-v_{i}$ and consider $K_{n-1}-F_{n-1}$. We shall show that $F_{n-1}$ is a subgraph of $K_{n-1}$ with the configuration $\mathcal{F}_{n-1}\left({ }^{*}\right)$.

Let $v_{j}$ be the neighbor of $v_{i}$ in $F_{n}$.

Case (1): $d_{F_{n-1}}\left(v_{j}\right) \geq 2$.

Clearly $F_{n-1}=\mathcal{F}_{n-1}\left({ }^{*}\right)$ if $i \geq j+3$ (otherwise $F_{n} \neq \mathcal{F}_{n}\left({ }^{*}\right)$ ). So assume that $i=j+2$.

If $d_{F_{n}}\left(v_{j+1}\right)=1$, then $v_{j}=v_{k-1}$ and we have $F_{n-1}=\mathcal{F}_{n-1}\left(^{*}\right)$ by definition.

If $d_{F_{n}}\left(v_{j+1}\right) \geq 2$, then $v_{j+1}=v_{k-1}$ (since $v_{j+2}=v_{i}$ is a pendant vertex). Hence $v_{j+1}$ is adjacent to some pendant vertex $v_{r}$ of $F_{n}$ where $r \in\{j+4, \ldots, n-1\}$.

Suppose $v_{j} v_{j+3} \notin E\left(F_{n-1}\right)$. Then $v_{j+3}$ is adjacent to some vertex $v_{s}$ where $s \in$ $\{0,1, \ldots, j-1\}$ with $d_{F_{n}}\left(v_{s}\right) \geq 2$. But this contradicts condition (iii) (in the definition of $\left.\mathcal{F}_{n}\left({ }^{*}\right)\right)$ since $v_{j+3} v_{s}$ crosses $v_{r} v_{j+1}$ and $s \neq j$.

Hence $v_{j} v_{j+3} \in E\left(F_{n-1}\right)$ and it follows that $F_{n-1}=\mathcal{F}_{n-1}\left(^{*}\right)$ in this case.

Case (2): $d_{F_{n-1}}\left(v_{j}\right)=1$.

In this case, $d_{F_{n}}\left(v_{j}\right)=2$. We assert that $j \in\{0, k-1\}$.

Suppose $1 \leq j \leq k-2$. Since $v_{j-1}, v_{j+1}$ are non-pendant vertices of $\mathcal{F}_{n}\left({ }^{*}\right)$, it follows that $v_{j-2}, v_{j+2}$ are the only neighbors of $v_{j}$ in $\mathcal{F}_{n}\left({ }^{*}\right)$. Assume without loss of generality that $v_{i}=v_{j+2}$. Hence $v_{j+1}=v_{k-1}$ is adjacent to a pendant vertex $v_{r}$ where $r \in\{j+4, \ldots, n-1\}$. Now the pendant vertex $v_{j+3}$ is adjacent to some non-pendant 
vertex $v_{s}$ where $s \in\{0,1, \ldots, j-1\}$. But then $v_{j+3} v_{s}, v_{r} v_{j+1}$ are crossing each other and $s \neq j$, a contradiction to condition (iii) in the definition of $\mathcal{F}_{n}\left({ }^{*}\right)$. This proves the assertion.

Assume without loss of generality that $j=k-1$. Then clearly $F_{n-1}=\mathcal{F}_{n-1}\left(^{*}\right)$.

By induction $K_{n-1}-\mathcal{F}_{n-1}\left({ }^{*}\right)$ admits no triangulation.

Assume on the contrary that $K_{n}-\mathcal{F}_{n}\left(^{*}\right)$ admits a triangulation $T_{n}$.

(i) Suppose $T_{n}$ contains $v_{i-1} v_{i+1}$. Then $v_{i}$ is a degree-2 vertex in $T_{n}$. Hence $T_{n}-v_{i}$ is a triangulation for $K_{n-1}-\mathcal{F}_{n-1}\left({ }^{*}\right)$, a contradiction.

(ii) Suppose $T_{n}$ does not contain $v_{i-1} v_{i+1}$. Then $v_{i} v_{t}$ is a diagonal in $T_{n}$ and $v_{i} v_{t}$ divides $K_{n}-F_{n}\left(^{*}\right)$ into $K_{m_{1}}-F_{m_{1}}$ and $K_{m_{2}}-F_{m_{2}}$ where $m_{1}+m_{2}-2=n$. Without loss of generality assume that $v_{i} v_{j}$ is an edge in $F_{m_{1}}$. Then it is readily checked that $\left.F_{m_{1}}=\mathcal{F}_{m_{1}}{ }^{*}\right)$. By induction $K_{m-1}-\mathcal{F}_{m_{1}}\left(^{*}\right)$ admits no triangulation, a contradiction.

This completes the proof.

The following result together with Proposition 1 characterize all graphs $F_{n}$ with at most $n-2$ edges such that $K_{n}-F_{n}$ admits a triangulation.

Theorem 2 Suppose $n \geq 6$ is a natural number and $F_{n}$ is a subgraph of the convex complete graph $K_{n}$ such that $\left|E\left(F_{n}\right)\right| \leq n-2$ and $F_{n}$ contains no boundary edges of $K_{n}$. Then $K_{n}-F_{n}$ admits a triangulation unless $F_{n}=\mathcal{F}_{n}\left({ }^{*}\right)$.

Proof: In view of Proposition 1, we assume that $F_{n} \neq \mathcal{F}_{n}\left(^{*}\right)$.

We prove the result by induction on $n$. The result is clearly true if $n=6$. Assume that $n \geq 7$ and the result is true for all convex graphs $K_{m}-F_{m}$ where $m<n$.

By Lemma 1, $K_{n}-F_{n}$ contains a vertex $v_{i}$ such that $v_{i-1} v_{i+1} \notin E\left(F_{n}\right)$.

Let $v_{i}$ which satisfies the condition in $(*)$ be such that $d_{F_{n}}\left(v_{i}\right)=k$ is maximal.

Delete the vertex $v_{i}$ from $K_{n}-F_{n}$. The resulting graph is a convex graph of the form $K_{n-1}-F_{n-1}$ where $F_{n-1}=F_{n}-v_{i}$ is a subgraph of $K_{n-1}$.

Case (1): $k \geq 2$.

Then $\left|E\left(F_{n-1}\right)\right| \leq n-4$. Clearly $F_{n-1}$ does not admit the configuration $\mathcal{F}_{n-1}\left({ }^{*}\right)$. By induction $K_{n-1}-F_{n-1}$ admits a triangulation $T_{n-1}$. As such $T_{n-1} \cup\left\{v_{i-1} v_{i}, v_{i} v_{i+1}\right\}$ is a triangulation for $K_{n}-F_{n}$.

Case (2): $k=1$.

Let $v_{j}$ be the neighbor of $v_{i}$ in $F_{n}$.

If $d_{F_{n}}\left(v_{j}\right)=1$, then $v_{j}$ is an isolated vertex in $F_{n-1}$ which implies that $K_{n-1}-F_{n-1}$ admits a triangulation $T_{n-1}$. Again $T_{n-1} \cup\left\{v_{i-1} v_{i}, v_{i} v_{i+1}\right\}$ is a triangulation for $K_{n}-F_{n}$.

Hence assume that $d_{F_{n}}\left(v_{j}\right) \geq 2$. 
If $F_{n-1} \neq \mathcal{F}_{n-1}\left({ }^{*}\right)$, then $K_{n-1}-F_{n-1}$ admits a triangulation $T_{n-1}$ by induction. Again $T_{n-1} \cup\left\{v_{i-1} v_{i}, v_{i} v_{i+1}\right\}$ is a triangulation for $K_{n}-F_{n}$.

Hence we assume that $F_{n-1}=\mathcal{F}_{n-1}\left(^{*}\right)$.

In view of the maximality on $d_{F_{n}}\left(v_{i}\right)=k$, we see that $v_{i-1}, v_{i+1}$ are both pendant vertices in $F_{n}$ (otherwise $F_{n}=\mathcal{F}_{n}\left({ }^{*}\right)$ ). Let $v_{s}$ and $v_{t}$ be the neighbors of $v_{i-1}$ and $v_{i+1}$ in $F_{n}$ respectively.

If $v_{i} v_{j}$ crosses neither $v_{i-1} v_{s}$ nor $v_{i+1} v_{t}$, then $F_{n}=\mathcal{F}_{n}\left({ }^{*}\right)$, a contradiction.

Hence we assume without loss of generality that $v_{i} v_{j}$ crosses $v_{i-1} v_{s}$ and that $s<j<i$.

If $|s-j|=1$, then we have $F_{n}=\mathcal{F}_{n}\left({ }^{*}\right)$, a contradiction.

If $|s-j|>1$, a triangulation for $K_{n}-F_{n}$ is given by the set of diagonals $v_{i-1} v_{j-1}$, $v_{i-1} v_{l}$ where $l \in\{j, j+1, \ldots, i-3\}=A$ and $v_{i} v_{m}$ for all $m \in\{0,1,2, \ldots, n-1\}-(A \cup$ $\{i-2, i-1, i, i+1\})$.

This completes the proof.

\section{Graphs with only $n-1$ edges}

We begin by describing a configuration for $F_{n}$ with $n$ vertices and $n-1$ edges such that $K_{n}-F$ admits no triangulation.

Definition 2 Let $F_{n}$ denote a subgraph of $K_{n}$ with no isolated vertices and having $n-1$ edges where $n \geq 5$. Let $\mathcal{J}_{n}\left(^{*}\right)$ denote a configuration of $F_{n}$ on $K_{n}$ such that (i) whenever $v_{i-1} v_{i+1} \notin E\left(\mathcal{J}_{n}\left({ }^{*}\right)\right)$, then $d_{F_{n}}\left(v_{i}\right) \leq 2$, and (ii) in the case that $d_{F_{n}}\left(v_{i}\right)=2$, then $\mathcal{J}_{n}\left(^{*}\right)-v_{i}=\mathcal{F}_{n-1}\left({ }^{*}\right)$ in $K_{n}-v_{i}$.

Suppose $k \in\{1,2\}$. Let $J_{n, k}$ denote the set of all degree- $k$ vertices in $\mathcal{J}_{n}\left(^{*}\right)$ satisfying the conditions (i) and (ii) in Definition 2. Note that if $v \in J_{n, 2}$, then no neighbor of $v$ is a pendant vertex in $\mathcal{J}_{n}\left({ }^{*}\right)$.

Definition 3 The configuration $\left.\mathcal{J}_{n}{ }^{*}\right)$ is said to be of Type-1 if $J_{n, 2}=\emptyset$ and there is an edge $e$ in $\mathcal{J}_{n}\left(^{*}\right)$ such that $\mathcal{J}_{n}\left({ }^{*}\right)-e$ is $\mathcal{F}_{n}\left({ }^{*}\right)$. The configuration $\mathcal{J}_{n}\left({ }^{*}\right)$ is said to be of Type-2 if (i) $J_{n, 2} \neq \emptyset$ and (ii) whenever $v_{i} \in J_{n, 2}$ and $v_{t}$ is a pendant vertex in $\mathcal{J}_{n}\left({ }^{*}\right)$ such that $v_{t} v_{s}$ crosses $v_{i} v_{j_{1}}$ and $v_{i} v_{j_{2}}$, then either $\left|j_{1}-s\right|=1$ or $\left|j_{2}-s\right|=1$.

Example 2 (i) Let $F$ be a subgraph of $K_{11}$ with vertex set $\left\{v_{0}, v_{1}, \ldots, v_{10}\right\}$ and edge set $\left\{v_{0} v_{2}, v_{0} v_{3}, v_{0} v_{7}, v_{0} v_{9}, v_{1} v_{3}, v_{1} v_{8}, v_{1} v_{10}, v_{2} v_{4}, v_{2} v_{6}, v_{3} v_{5}\right\}$. Then $F$ is an example of a $\mathcal{J}_{11}\left(^{*}\right)$ configuration in $K_{11}$. Here $J_{11,1}=\left\{v_{5}, v_{6}, \ldots, v_{9}\right\}$ and $J_{11,2}=\emptyset$. Hence $F$ is of Type-1. Note that $\mathcal{J}_{11}\left(^{*}\right)-\left\{v_{0} v_{3}\right\} \cong \mathcal{F}_{11}\left(^{*}\right)$. 
(ii) Let $F$ be a subgraph of $K_{11}$ with vertex set $\left\{v_{0}, v_{1}, \ldots, v_{10}\right\}$ and edge set $\left\{v_{0} v_{2}, v_{0} v_{7}\right.$, $\left.v_{0} v_{9}, v_{1} v_{3}, v_{1} v_{8}, v_{1} v_{10}, v_{2} v_{4}, v_{2} v_{6}, v_{3} v_{5}, v_{5} v_{9}\right\}$. Then $F$ is an example of a $\mathcal{J}_{11}\left({ }^{*}\right)$ configuration in $K_{11}$. Here $J_{11,1}=\left\{v_{6}, v_{7}, v_{8}\right\}$ and $J_{11,2}=\left\{v_{5}, v_{9}\right\}$. Hence $F$ of Type-2.

We shall define three $\mathcal{J}_{6}\left({ }^{*}\right)$ configurations $J_{1}, J_{2}, J_{3}$ (on $K_{6}$ ) each of Type-2 with $J_{6,1}=\emptyset$. Let $J_{1}=v_{1} v_{4} v_{2} v_{5} v_{1} \cup\left\{v_{0} v_{3}\right\}, J_{2}=v_{1} v_{3} v_{5} v_{1} \cup\left\{v_{0} v_{2}, v_{1} v_{4}\right\}$ and $J_{3}=v_{1} v_{3} v_{5} v_{1} \cup$ $v_{2} v_{0} v_{4}$.

Lemma 2 Suppose $F_{6}$ is a Type-2 $\mathcal{J}_{6}\left({ }^{*}\right)$. Then $J_{6,1}=\emptyset$ if and only if $F_{6}=J_{i}$ for some $i \in\{1,2,3\}$. Moreover $K_{6}-J_{i}$ admits no triangulation for any $i=1,2,3$.

Proof: The sufficiency is clear. We shall prove the necessity.

Note that, for any $v \in J_{6,2}, \mathcal{J}_{6}\left(^{*}\right)-v=\mathcal{F}_{5}\left({ }^{*}\right)$. Note also that there is only one configuration of $\mathcal{F}_{5}\left({ }^{*}\right)$ on $K_{5}$ which is given by $E\left(\mathcal{F}_{5}\left({ }^{*}\right)\right)=\left\{x_{0} x_{2}, x_{1} x_{3}, x_{1} x_{4}\right\}$.

Now, there are only three possible ways to place $v$ on the boundary edge of $K_{5}$ (in order to recover $\mathcal{J}_{6}\left({ }^{*}\right)$ from $\left.\mathcal{F}_{5}\left({ }^{*}\right)\right)$. Without loss of generality, assume that $v$ lies on the edge $x_{i} x_{i+1}$ for some $i \in\{1,2,3\}$. In each case, we join $v$ to two vertices of $\mathcal{F}_{5}\left({ }^{*}\right)$ subject to the condition that $J_{6,1}=\emptyset$. We then arrive at the conclusion that $F_{6}=J_{i}$, $i \in\{1,2,3\}$.

Suppose on the contrary that $K_{6}-J_{i}$ admits a triangulation $T_{i}$. For any $v_{j} \in J_{6,2}$, the edge $v_{j-1} v_{j+1}$ is not a diagonal edge in $T_{i}$ (because $J_{i}-v_{j}=\mathcal{F}_{5}\left({ }^{*}\right)$ ). Hence $v_{j} v_{t}$, for some vertex $v_{t}$ (not adjacent to $v_{j}$ ) in $J_{i}$, is a diagonal edge in $T_{i}$. But then it is routine to check that $v_{j} v_{t}$ cannot be combined with another two non-adjacent edges in $J_{i}$ to form the set of diagonal edges of $T_{i}$, a contradiction.

Lemma 3 Suppose $F_{n}$ is a Type-2 $\mathcal{J}_{n}\left({ }^{*}\right)$ configuration and $n \geq 7$, If $J_{n, 1}=\emptyset$, then for each $v_{i} \in J_{n, 2}, v_{i-2} v_{i}, v_{i} v_{i+2} \in E\left(\mathcal{J}_{n}\left({ }^{*}\right)\right)$.

Proof: Since $v_{i} \in J_{n, 2}$, we have $\mathcal{J}_{n}\left(^{*}\right)-v_{i}=\mathcal{F}_{n-1}\left(^{*}\right)$. Let $u_{0}, u_{1}, \ldots, u_{n-2}$ denote the vertices of $\mathcal{F}_{n-1}\left({ }^{*}\right)$ arranged in cyclic order.

Now, $J_{n, 1}=\emptyset$ implies that $\mathcal{F}_{n-1}\left({ }^{*}\right)$ has at least two pendant vertices and at most three pendant vertices $u_{j}$ such that $u_{j-1} u_{j+1} \notin E\left(\mathcal{F}_{n-1}\left({ }^{*}\right)\right)$. Moreover such pendant vertices must be in consecutive order (in $\mathcal{F}_{n-1}\left({ }^{*}\right)$ ).

Now, insert the vertex $v_{i}$ into $\mathcal{F}_{n-1}\left({ }^{*}\right)$ to recover $\mathcal{J}_{n}\left(^{*}\right)$. Since $n \geq 7$ and $J_{n, 1}=\emptyset, v_{i}$ must be inserted in between two pendant vertices of $\mathcal{F}_{n-1}\left({ }^{*}\right)$.

If there are only two such pendant vertices $u_{j}$ and $u_{j+1}$, then either $u_{j}=v_{i-1}, u_{j+1}=$ $v_{i+1}$ or else $u_{j}=v_{i+1}, u_{j-1}=v_{i-1}$. Either case implies that $v_{i-2} v_{i}, v_{i} v_{i+2} \in E\left(\mathcal{J}_{n}\left({ }^{*}\right)\right)$.

If there are three such pendant vertices $u_{j-1}, u_{j}$ and $u_{j+1}$, then again either $u_{j}=$ $v_{i-1}, u_{j+1}=v_{i+1}$ or else $u_{j}=v_{i+1}, u_{j-1}=v_{i-1}$ and we have the same conclusion as before.

This proves the lemma. 
Proposition 2 Suppose $F_{n}$ is a subgraph of the convex complete graph $K_{n}$ with a Type-1 or Type-2 configuration $\mathcal{J}_{n}\left({ }^{*}\right)$. Then $K_{n}-\mathcal{J}_{n}\left({ }^{*}\right)$ admits no triangulation for any natural number $n \geq 5$.

Proof: We prove this by induction on $n$. The result is clearly true if $n=5$. Hence we assume that $n \geq 6$ and that the result is true for all convex graphs $K_{m}-F_{m}$ where $m<n$.

By Lemma 1, $K_{n}-\mathcal{J}_{n}\left(^{*}\right)$ contains a vertex $v_{j}$ such that $v_{j-1} v_{j+1} \notin E\left(\mathcal{J}_{n}\left({ }^{*}\right)\right)$. By the definition of $\mathcal{J}_{n}\left({ }^{*}\right)$, we see that $d_{\mathcal{J}_{n}\left({ }^{*}\right)}\left(v_{j}\right) \leq 2$.

Case (1): $\mathcal{J}_{n}\left(^{*}\right)$ is of Type-1.

In this case $d_{\mathcal{J}_{n}\left({ }^{*}\right)}\left(v_{j}\right)=1$, and there is an edge $e \in \mathcal{J}_{n}\left({ }^{*}\right)$ satisfying $\mathcal{J}_{n}\left({ }^{*}\right)-e \cong$ $\mathcal{F}_{n}\left({ }^{*}\right)$. Since $K_{n}-\mathcal{F}_{n}\left({ }^{*}\right)$ admits no triangulation, it follows that $K_{n}-\mathcal{J}_{n}\left(^{*}\right)$ admits no triangulation

Case (2): $\mathcal{J}_{n}\left(^{*}\right)$ is of Type-2.

(a) Suppose $J_{n, 1} \neq \emptyset$.

In this case, let $v_{j}$ which satisfies the condition $v_{j-1} v_{j+1} \notin E\left(\mathcal{J}_{n}\left({ }^{*}\right)\right)$ be chosen such that $d_{\mathcal{J}_{n}\left({ }^{*}\right)}\left(v_{j}\right)=1$.

Then $\mathcal{J}_{n}\left({ }^{*}\right)-v_{j}=\mathcal{J}_{n-1}\left(^{*}\right)$ in $K_{n-1}$. By induction, $K_{n-1}-\mathcal{J}_{n-1}\left(^{*}\right)$ admits no triangulation.

Assume on the contrary that $K_{n}-\mathcal{J}_{n}\left(^{*}\right)$ admits a triangulation $T_{n}$.

Suppose $T_{n}$ contains $v_{j-1} v_{j+1}$. Then $T_{n}-v_{j}$ is a triangulation for $K_{n-1}-\mathcal{J}_{n-1}\left(^{*}\right)$, a contradiction.

Hence assume that $T_{n}$ does not contain $v_{j-1} v_{j+1}$. Then $T_{n}$ contains a diagonal $v_{j} v_{t}$. Let $v_{j_{1}}$ be the neighbor of $v_{j}$ in $\mathcal{J}_{n}\left(^{*}\right)$.

Clearly $v_{j} v_{t}$ divides $K_{n}-\mathcal{J}_{n}\left(^{*}\right)$ into two convex graphs $K_{m_{1}}-F_{m_{1}}$ and $K_{m_{2}}-F_{m_{2}}$ where $m_{1}+m_{2}=n+2$. Without loss of generality assume that $v_{j} v_{j_{1}}$ is an edge in $F_{m_{1}}$. Note that $K_{m_{2}}-F_{m_{2}}$ admits a triangulation (since $v_{j}$ is an isolated vertex in $F_{m_{2}}$ ).

If $J_{n, 2} \cap V\left(F_{m_{1}}\right)=\emptyset$, then $F_{m_{1}}=\mathcal{F}_{m_{1}}\left({ }^{*}\right)$ which admits no triangulation by Proposition 1. Hence we assume that $J_{n, 2} \cap V\left(F_{m_{1}}\right)$ contains a vertex $v_{i}$.

(i) If both neighbors of $v_{i}$ are in $V\left(F_{m_{1}}\right)$, then $F_{m_{1}}$ is $\mathcal{J}_{m_{1}}\left(^{*}\right)$. By induction, $K_{m_{1}}-F_{m_{1}}$ admits no triangulation and this implies that $K_{n}-\mathcal{J}_{n}\left({ }^{*}\right)$ admits no triangulation, a contradiction.

(ii) If only one of the neighbors of $v_{i}$ is in $V\left(F_{m_{1}}\right)$, then $F_{m_{1}}$ is $\mathcal{F}_{m_{1}}\left(^{*}\right)$ and again we have a contradiction.

(iii) If both neighbors $v_{i_{1}}, v_{i_{2}}$ of $v_{i}$ are in $V\left(F_{m_{2}}\right)$, then the edge $v_{j} v_{s}$ (incident to the pendant vertex $v_{j}$ ) crosses both the edges $v_{i} v_{i_{1}}, v_{i} v_{i_{2}}$ with $\left|s-i_{1}\right|>1$ and $\left|s-i_{2}\right|>1$, a contradiction to the definition of Type- $2 \mathcal{J}_{n}\left({ }^{*}\right)$ configuration.

(b) Suppose $J_{n, 1}=\emptyset$. 
Then $v_{j} \in J_{n, 2}$ and $\mathcal{J}_{n}\left({ }^{*}\right)-v_{j}=\mathcal{F}_{n-1}\left(^{*}\right)$ in $K_{n-1}$. By Proposition 1, $K_{n-1}-\mathcal{F}_{n-1}\left(^{*}\right)$ admits no triangulation.

When $n=6, K_{6}-\mathcal{J}_{6}\left({ }^{*}\right)$ admits no triangulation by Lemma 2 ,

When $n \geq 7$, assume on the contrary that $K_{n}-\mathcal{J}_{n}\left(^{*}\right)$ admits a triangulation $T_{n}$.

If $T_{n}$ contains $v_{j-1} v_{j+1}$, then $T_{n}-v_{j}$ is a triangulation for $K_{n-1}-\mathcal{F}_{n-1}\left(^{*}\right)$, a contradiction.

Hence assume that $T_{n}$ does not contain $v_{j-1} v_{j+1}$. Then $T_{n}$ contains a diagonal $v_{j} v_{t}$.

By Lemma 3, $v_{j-2} v_{j}, v_{j} v_{j+2} \in E\left(\mathcal{J}_{n}\left(^{*}\right)\right)$ (since $\left.n \geq 7\right)$.

Clearly $v_{j} v_{t}$ divides $K_{n}-\mathcal{J}_{n}\left(^{*}\right)$ into two convex graphs $K_{m_{1}}-F_{m_{1}}$ and $K_{m_{2}}-F_{m_{2}}$ where $m_{1}+m_{2}-2=n$ with $F_{m_{1}}$ and $F_{m_{2}}$ containing $v_{j} v_{j-2}$ and $v_{j} v_{j+2}$ respectively.

Note that $v_{t} \notin J_{n, 2}$. This is because the vertex $v_{j}$ in $\mathcal{J}_{n}\left({ }^{*}\right)-v_{t}$ is a non-pendant vertex and $v_{j-1} v_{j+1}$ is not an edge of $\mathcal{J}_{n}\left({ }^{*}\right)-v_{t}$ implying that $\mathcal{J}_{n}\left({ }^{*}\right)-v_{t} \neq \mathcal{F}_{n-1}\left(^{*}\right)$.

If $V\left(F_{m_{1}}\right) \cap J_{n, 2}=\emptyset$, then clearly $F_{m_{1}}$ is $\left.\mathcal{F}_{m_{1}}{ }^{*}\right)$. By Proposition 1, $\left.K_{m_{1}}-\mathcal{F}_{m_{1}}{ }^{*}\right)$ admits no triangulation and this implies that $K_{n}-\mathcal{J}_{n}\left({ }^{*}\right)$ admits no triangulation, a contradiction.

Hence assume that there exists $v_{i} \in V\left(F_{m_{1}}\right) \cap J_{n, 2}$. Clearly, $v_{i}$ is adjacent to $v_{j}$ in $\mathcal{J}_{n}\left(^{*}\right)$ (otherwise, the vertex $v_{j}$ in $\mathcal{J}_{n}\left(^{*}\right)-v_{i}$ is a non-pendant vertex with $v_{j-1} v_{j+1} \notin$ $\left(\mathcal{J}_{n}\left({ }^{*}\right)-v_{i}\right)$, and this implies that $\mathcal{J}_{n}\left(^{*}\right)-v_{i} \neq \mathcal{F}_{n-1}\left({ }^{*}\right)$, a contradiction). Thus $i=j-2$.

We assert that $\left(F_{m_{2}}-v_{j}\right) \cap J_{n, 2}=\emptyset$. To see this, suppose $v_{r} \in\left(F_{m_{2}}-v_{j}\right) \cap J_{n, 2}$. By the same argument in the preceding paragraph (on $v_{i}$ ), it follows that $v_{r}$ is adjacent to $v_{j}$ and hence $v_{r}=v_{j+2}$. Moreover $v_{r}$ is not adjacent to $v_{i}$ (since $n \geq 7$ ). As such, $\mathcal{J}_{n}\left({ }^{*}\right)-v_{r}$ contains $v_{i}$ with $v_{i-1} v_{i+1} \notin \mathcal{J}_{n}\left(^{*}\right)-v_{r}$ (and $v_{i}$ is non-pendant) and this implies that $\mathcal{J}_{n}\left(^{*}\right)-v_{i} \neq \mathcal{F}_{n-1}\left({ }^{*}\right)$, a contradiction.

It follows from the assertion that $F_{m_{2}}=\mathcal{F}_{m_{2}}\left(^{*}\right)$. By Proposition 1, $K_{m_{2}}-\mathcal{F}_{m_{2}}\left(^{*}\right)$ admit no triangulation and this implies that $K_{n}-\mathcal{J}_{n}\left({ }^{*}\right)$ admits no triangulation, a contradiction.

This completes the proof.

Theorem 3 Suppose $n \geq 5$ is a natural number and $F_{n}$ is a subgraph of the convex complete graph $K_{n}$ such that $\left|E\left(F_{n}\right)\right|=n-1$ and $F_{n}$ contains no boundary edges of $K_{n}$. Then $K_{n}-F_{n}$ admits a triangulation unless $F_{n}$ is Type-1 or Type-2 $\mathcal{J}_{n}\left({ }^{*}\right)$.

Proof: We prove the result by induction on $n$.

The result is clearly true if $n=5,6$. Assume that $n \geq 7$ and that the result is true for all convex graphs $K_{m}-F_{m}$ where $m<n$.

By Lemma 1, $K_{n}-F_{n}$ contains a vertex $v_{i}$ such that $v_{i-1} v_{i+1} \notin E\left(F_{n}\right)$.

Let $v_{i}$ which satisfies the condition in $(*)$ be such that $d_{F_{n}}\left(v_{i}\right)=k$ is maximal. 
Delete the vertex $v_{i}$ from $K_{n}-F_{n}$. The resulting graph is a convex graph of the form $K_{n-1}-F_{n-1}$ where $F_{n-1}=F_{n}-v_{i}$ is a subgraph of $K_{n-1}$.

Case (1): $k \geq 3$.

Then $\left|E\left(F_{n-1}\right)\right| \leq n-4$. By Theorem 2 , $K_{n-1}-F_{n-1}$ admits a triangulation $T_{n-1}$. As such $T_{n-1} \cup\left\{v_{i-1} v_{i}, v_{i} v_{i+1}\right\}$ is a triangulation for $K_{n}-F_{n}$.

Case (2): $k=2$.

If $F_{n-1} \neq \mathcal{F}_{n-1}\left(^{*}\right)$, then $K_{n-1}-F_{n-1}$ admits a triangulation $T_{n-1}$ by Theorem 2 . Again $T_{n-1} \cup\left\{v_{i-1} v_{i}, v_{i} v_{i+1}\right\}$ is a triangulation for $K_{n}-F_{n}$.

Hence we assume that $\left.F_{n-1}=\mathcal{F}_{n-1}{ }^{*}\right)$.

Let $v_{j_{1}}$ and $v_{j_{2}}$ be the neighbors of $v_{i}$ in $F_{n}$.

Now, there is an edge $v_{t} v_{s}$ incident to a pendant vertex $v_{t}$ such that $v_{t} v_{s}$ crosses both the edges $v_{i} v_{j_{1}}, v_{i} v_{j_{1}}$ with $\left|j_{1}-s\right|>1$ and $\left|j_{2}-s\right|>1$ (otherwise $F_{n}$ is Type- $2 \mathcal{J}_{n}\left({ }^{*}\right)$ ).

We can assume without loss of generality that $s<j_{1}<j_{2}<i$.

Let $G_{1}$ and $G_{2}$ be subgraphs of $K_{n}-F_{n}$ induced by the vertices $\left\{v_{j_{1}-1}, v_{j_{1}}, \ldots, v_{i-1}\right\}$ and $\left\{v_{i-1}, v_{i}, \ldots, v_{n-1}, v_{0}, \ldots, v_{j_{1}-1}\right\}$ respectively (with $v_{t}$ contained in $G_{1}$ ). Clearly, $d_{G_{1}}\left(v_{t}\right)=0$ and $d_{G_{2}}\left(v_{i}\right)=0$ which imply that $G_{i}$ admits a triangulation $T_{i}, i=1,2$. As such, $T=T_{1} \cup T_{2}$ is a triangulation for $K_{n}-F_{n}$.

Case (3): $k=1$.

Let $v_{j}$ be the neighbor of $v_{i}$ in $F_{n}$.

Then $d_{F_{n}}\left(v_{j}\right) \geq 2$ otherwise $K_{n-1}-F_{n-1}$ admits a triangulation $T_{n-1}$ (because $v_{j}$ is an isolated vertex in $\left.K_{n-1}-F_{n-1}\right)$ which means that $T_{n-1} \cup\left\{v_{i-1} v_{i}, v_{i} v_{i+1}\right\}$ is a triangulation for $K_{n}-F_{n}$.

By the maximality of $k$, we have $v_{j-1} v_{j+1} \in E\left(F_{n}\right)$.

Now, $\left|E\left(F_{n-1}\right)\right|=n-2$. If $F_{n-1}$ is not the configuration $\mathcal{J}_{n-1}\left({ }^{*}\right)$, then $K_{n-1}-F_{n-1}$ admits a triangulation $T_{n-1}$ by induction. Again $T_{n-1} \cup\left\{v_{i-1} v_{i}, v_{i} v_{i+1}\right\}$ is a triangulation for $K_{n}-F_{n}$.

Hence we assume that $F_{n-1} \cong \mathcal{J}_{n-1}\left({ }^{*}\right)$.

(i) Suppose $\mathcal{J}_{n-1}\left(^{*}\right)$ is of Type-2.

Then there is a unique degree-2 vertex $v_{s}$ in $\mathcal{J}_{n-1}\left({ }^{*}\right)$ such that $\mathcal{J}_{n-1}\left({ }^{*}\right)-v_{s}=\mathcal{F}_{n-2}\left(^{*}\right)$ (in $K_{n-2}=K_{n}-\left\{v_{i}, v_{s}\right\}$ ). It is easy to see that either $v_{s}=v_{i+1}$ or $v_{s}=v_{i-1}$. Assume without loss of generality that $v_{s}=v_{i+1}$. Hence $v_{i-1} v_{s+1}$ is not an edge in $\mathcal{J}_{n-1}\left(^{*}\right)$ (since $\left.v_{s} \in J_{n-1,2}\right)$.

In view of maximality of $k, v_{s-1} v_{s+1} \in E\left(F_{n}\right)$. Since $v_{i}=v_{s-1}$ is a pendant vertex, it follows that $v_{j}=v_{s+1}$.

Clearly, $v_{i-1}$ is not adjacent to $v_{j}=v_{s+1}$. Hence $v_{i-1} v_{j}$ is a boundary edge in $K_{n-2}-\mathcal{F}_{n-2}\left(^{*}\right)$. 
Let $v_{s_{1}}, v_{s_{2}}$ be the neighbors of $v_{s}$ in $\mathcal{J}_{n-1}\left({ }^{*}\right)$.

Since $v_{j-1} v_{j+1}=v_{s} v_{s+2}$ is an edge in $F_{n}$, we may assume that $v_{s+2}=v_{s_{1}}$, and hence $s_{2} \geq s+3$.

By the maximality of $k$ we see that $v_{i-1}$ is a pendant vertex in $F_{n}$ (since $v_{i}$ is a pendant vertex and $v_{i}$ is adjacent only to $v_{j}=v_{s+1}$ ). But this means that $v_{i-1}$ is also a pendant vertex in $\left.\mathcal{F}_{n-2}{ }^{*}\right)$.

Note that $v_{s_{1}}$ is a non-pendant vertex in $F_{n}$ (otherwise $\mathcal{J}_{n-1}\left({ }^{*}\right)-v_{s}$ contains an isolated vertex $v_{s_{1}}$, a contradiction).

It is easy to see that $\mathcal{F}_{n-2}\left({ }^{*}\right) \cup\left\{v_{s} v_{s_{1}}\right\} \cup\left\{v_{i} v_{j}\right\}=\mathcal{F}_{n}\left({ }^{*}\right)$. But then $\mathcal{F}_{n}\left({ }^{*}\right) \cup\left\{v_{s} v_{s_{2}}\right\}=F_{n}$ implying that $F_{n}$ is a $\mathcal{J}_{n}\left(^{*}\right)$ configuration of Type-1, a contradiction.

(ii) Suppose $\mathcal{J}_{n-1}\left(^{*}\right)$ is of Type- 1 .

Then there is an edge $e$ in $\mathcal{J}_{n-1}\left({ }^{*}\right)$ such that $\mathcal{J}_{n-1}\left(^{*}\right)-e$ is $\mathcal{F}_{n-1}\left(^{*}\right)$.

We assert that $v_{i-1}, v_{i+1}$ are both pendant vertices in $F_{n}$.

To see this suppose $v_{i-1}$ is a non-pendant vertex in $\mathcal{F}_{n-1}\left({ }^{*}\right)$. Then it follows from the maximality of $k$ that $v_{i} v_{i-2}=v_{i} v_{j}$ is an edge of $F_{n}$. But this means that $\left.\mathcal{F}_{n-1}{ }^{*}\right) \cup$ $\left\{v_{i} v_{j}\right\}=\mathcal{F}_{n}\left({ }^{*}\right)$ implying that $F_{n}$ is a $\mathcal{J}_{n}\left(^{*}\right)$ configuration of Type-1 (because $\mathcal{F}_{n-1}\left({ }^{*}\right) \cup$ $\left\{v_{i} v_{j}\right\} \cup e=F_{n}$ ), a contradiction.

Hence let $v_{r}$ and $v_{s}$ be the neighbors of $v_{i-1}$ and $v_{i+1}$ in $F_{n}$ respectively.

Suppose first that either (a) $v_{i} v_{j}$ crosses neither $v_{i-1} v_{r}$ nor $v_{i+1} v_{s}$ or that (b) $v_{i} v_{j}$ crosses $v_{i-1} v_{r}$ (or $v_{i+1} v_{s}$ ) with $|j-r|=1$ (or $|j-s|=1$ ). In any of these cases we have $\mathcal{F}_{n-1}\left({ }^{*}\right) \cup\left\{v_{i} v_{j}\right\}=\mathcal{F}_{n}\left({ }^{*}\right)$ implying that $F_{n}$ is a $\mathcal{J}_{n}\left({ }^{*}\right)$ configuration of Type- 1 , a contradiction.

Hence assume without loss of generality that $v_{i} v_{j}$ crosses $v_{i-1} v_{r}$ with $|j-r|>1$. In this case, let $G_{1}$ and $G_{2}$ be the subgraphs of $K_{n}-F_{n}$ induced by the vertices $\left\{v_{j-1}, v_{j}, \ldots, v_{i-1}\right\}$ and $\left\{v_{i-1}, v_{i}, \ldots, v_{j-1}\right\}$ respectively. Clearly $v_{i-1}$ and $v_{i}$ are isolated vertex in $G_{1}$ and $G_{2}$ respectively. Hence $G_{i}$ admits a triangulation $T_{i}$ for each $i=1,2$. A triangulation of $K_{n}-F_{n}$ is given by $T=T_{1} \cup T_{2}$.

This completes the proof.

\section{Potentially triangulable graphs}

We now look at the possibility of packing a graph $F$ with $n$ vertices and $n$ edges in the convex complete graph $K_{n}$ so that $K_{n}-F$ admits a triangulation. We shall confine our attention to the case where $F_{n}$ is a 2-regular graph. We begin with the following example.

Example 3 Suppose $F$ is a 7-cycle. If $F$ is of the form $v_{0} v_{2} v_{4} v_{6} v_{1} v_{3} v_{5} v_{0}$ or of the form $v_{0} v_{3} v_{6} v_{2} v_{5} v_{1} v_{4} v_{0}$ in $K_{7}$. Then it is easy to see that $K_{7}-F$ admits no triangulation. On 
the other hand, if $F$ is of the form $v_{0} v_{2} v_{6} v_{4} v_{1} v_{3} v_{5} v_{0}$, then $K_{7}-F$ admits a triangulation whose diagonals are $v_{6} v_{1}, v_{1} v_{5}, v_{5} v_{2}, v_{2} v_{4}$.

Definition 4 Let $K_{n}$ be a convex complete graph with $n$ vertices. $F$ is said to be potentially triangulable in $K_{n}$ if there exists a configuration of $F$ in $K_{n}$ such that $K_{n}-F$ admits a triangulation.

Theorem 4 Suppose $F_{n}$ is an n-cycle. Then $F_{n}$ is potentially triangulable in $K_{n}$ if and only if $n \geq 7$.

Proof: It is easy to see that $K_{n}-F_{n}$ admits no triangulation if $n \leq 5$.

Suppose $K_{6}-F_{6}$ admits a triangulation $T$. Since $T$ has precisely 3 diagonals, there is a vertex $v_{i}$ which is incident with 2 diagonals of $T$. But this is clearly a contradiction since $K_{6}-\left(F_{6} \cup v_{0} v_{1} v_{2} \cdots v_{5} v_{0}\right)$ is 1-regular.

For $n=7, F_{n}$ is potentially triangulable in $K_{n}$ by Example 3 ,

For the rest of the proof, we assume that $n \geq 8$.

Case (1): $n$ is even.

Note that $n$ can be written as $n=2^{s} t$ for some positive natural number $s$ and some positive odd natural number $t$. Let $\alpha=t+2$. Then $\operatorname{gcd}(t, \alpha)=1$.

By relabeling the vertices if necessary, we may assume without loss of generality that $F_{n}$ takes the form

$$
v_{0} v_{\alpha} v_{2 \alpha} v_{3 \alpha} \ldots v_{(n-1) \alpha} v_{0}
$$

Here the operation is reduced modulo $n$. That is, the edges of $F_{n}$ are of the form $v_{i} v_{j}$ where $j-i \in S_{n}=\{t+2, n-t-2\}$.

The subgraph $G^{\prime}$ induced by the vertices $v_{0}, v_{2}, v_{4}, \ldots, v_{n-2}$ is a convex complete graph $K_{n / 2}$ (because it contains no edges from $F_{n}$ ). As such $G^{\prime}$ admits a triangulation $T^{\prime}$ (by Theorem 2). By adding the vertices $v_{1}, v_{3}, \ldots, v_{n-1}$ to $T^{\prime}$ together with the edges $v_{0} v_{1} v_{2} v_{3} \ldots v_{n-1} v_{0}$, we have a triangulation of $K_{n}-F_{n}$.

Case (2): $n \geq 9$ is odd.

Let $\alpha=\lfloor n / 2\rfloor$ and let $F_{n}$ take the form

$$
v_{0} v_{\alpha} v_{2 \alpha} v_{3 \alpha} \ldots v_{(n-1) \alpha} v_{0} .
$$

Here again, the operation is reduced modulo $n$. That is, the edges of $F_{n}$ are of the form $v_{i} v_{j}$ where $j-i \in S_{n}=\{(n-1) / 2,(n+1) / 2\}$.

Let $\beta=\lceil n / 3\rceil$. Consider the subgraphs $G_{0}, G_{1}, G_{2}$ induced by the sets of vertices $\left\{v_{0}, v_{1}, v_{2}, \ldots, v_{\beta}\right\},\left\{v_{\beta}, v_{\beta+1}, v_{\beta+2}, \ldots, v_{2 \beta}\right\}$ and $\left\{v_{2 \beta}, v_{2 \beta+1}, \ldots, v_{0}\right\}$ respectively. Then $G_{i}, i=0,1,2$ is a convex complete subgraph of $K_{n}-F_{n}$ (because it contains no edges from $F_{n}$ ). Hence $G_{i}$ admits a triangulation $T_{i}, i=0,1,2$. As such, $T_{0} \cup T_{1} \cup T_{2}$ is a triangulation of $K_{n}-F_{n}$. 
Remark 1 Let $K_{n}$ be a convex complete graph with $n$ vertices and let $F$ be a union of $k$ disjoint cycles in $K_{n}$ where $|V(F)|=n-3 \geq 4$. It is easy to see that one can place these $k$ cycles in $K_{n}$ such that (i) no edge of $F$ is a boundary edge of $K_{n}$, and (ii) there are three vertices $x, y, z \in V\left(K_{n}\right)-V(F)$ such that $x y$ is a boundary edge of $K_{n}$ and $x, y, z$ separate $V(F)$ into two sets. Figure 1 illustrates an example where $n=15, k=3$ and $F=C_{4} \cup C_{3} \cup C_{5}$. Here the cycles are drawn with dashed lines. By Theorem Q, $K_{n}-F$ admits a triangulation (because $|E(F)|=n-3$ ).

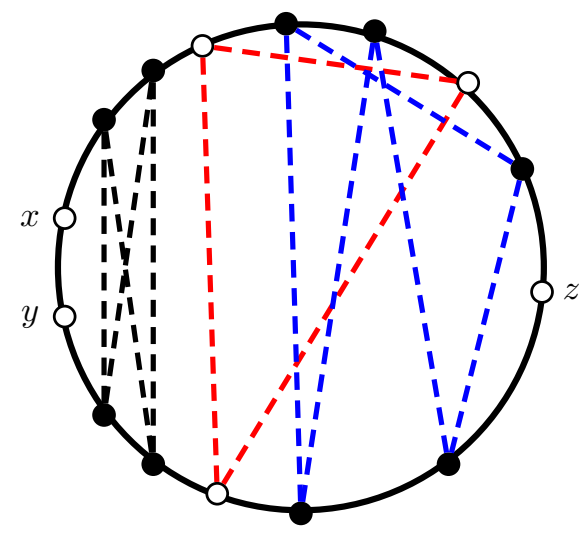

Figure 1: A drawing of $K_{15}-\left(C_{4} \cup C_{3} \cup C_{5}\right)$.

Theorem 5 Let $F_{n}$ be a 2-regular graph with $n$ vertices. Then $F_{n}$ is potentially triangulable in $K_{n}$ if and only if $F_{n} \notin\left\{C_{3}, C_{4}, C_{5}, C_{6}, C_{3} \cup C_{3}, C_{3} \cup C_{4}\right\}$.

Proof: If $F_{n}$ is connected, the result is true by Theorem 4. Hence we assume that $F_{n}$ is a union of disjoint cycles.

Let the vertices of the convex complete graph $K_{n}$ be denoted by $v_{0}, v_{1}, \ldots, v_{n-1}$.

Since $K_{6}-\left(C_{3} \cup C_{3} \cup v_{0} v_{1} v_{2} \cdots v_{5} v_{0}\right)$ is 1-regular, it follows that $K_{n}-F_{n}$ admits no triangulation if $F_{n}=C_{3} \cup C_{3}$.

Suppose $F_{n}=C_{3} \cup C_{4}$ and assume that that $K_{n}-F_{n}$ admits a triangulation $T$. Since $K_{n}-F_{n}$ is a 2-regular graph, the graph $D$ induced by the the diagonal edges of $T$ is a path with 5 vertices. Assume without loss of generality that $D=v_{1} v_{6} v_{2} v_{5} v_{3}$. It follows that $v_{0} v_{2} v_{4} v_{0} \subset F_{n}$. Consequently $E\left(C_{4}\right) \cap E(D) \neq \emptyset$, a contradiction.

For the rest of the proof, we assume that $n \geq 8$. Let $C$ be a cycle in $F_{n}$ and let $|V(C)|=p$. Also let $F^{*}=F_{n}-C$.

Case (1): $p \geq 8$. 
(i) Suppose $p$ is even. Use the method in Case (1) of the proof of Theorem 4 to construct a triangulation $T_{p}$ of $K_{p}-C$. To obtain a triangulation for $K_{n}-F_{n}$, we do the following.

Assume first that $\left|V\left(F^{*}\right)\right| \geq 4$. Insert $n-p$ vertices into the edges $v_{0} v_{1}, v_{1} v_{2}$ so that these $n-p$ vertices together with $v_{0}, v_{1}, v_{2}$ form a convex complete graph $K_{n-p+3}$. Also, join each of these $n-p$ vertices to every vertex in $K_{p}$. We then place all the disjoint cycles $F^{*}$ in $K_{n-p+3}$ in the same manner as was done in Remark 1 (with $v_{0}, v_{2}, v_{1}$ playing the roles of $x, y, z$ respectively) and obtain a triangulation $T^{*}$ for $K_{n-p+3}-F^{*}$. Consequently, $T_{p} \cup T^{*}$ is a triangulation for $K_{n}-F_{n}$.

Now assume that $F^{*}$ is a 3 -cycle. Insert 3 vertices $u, v, w$ into the edges $v_{0} v_{1}, v_{1} v_{2}, v_{2} v_{3}$ respectively so that $\left\{v_{0}, u, v_{1}, v, v_{2}, w, v_{3}\right\}$ becomes the vertex set of a convex complete graph $K_{7}$. Also, join $u, v, w$ each to every vertex of $K_{p}$. Take $u v w u$ to be the 3 -cycle $F^{*}$. Then $T_{p} \cup\left\{v_{0} u v_{1} v v_{2} w v_{3}\right\}$ is a triangulation for $K_{n}-F_{n}$.

(ii) Suppose $p$ is odd. Use the method in Case (2) of the proof of Theorem 4 to construct a triangulation $T_{p}$ of $K_{p}-C$. Recall that $T_{p}=T_{0} \cup T_{1} \cup T_{2}$ where $T_{i}$ is a triangulation of $G_{i}, i=0,1,2$. To obtain a triangulation for $K_{n}-F_{n}$, we do the following.

As before we first assume that $\left|V\left(F^{*}\right)\right| \geq 4$. Insert $n-p$ vertices into the edges $v_{0} v_{1}, v_{1} v_{2}$ so that these $n-p$ vertices together with $v_{0}, v_{1}, v_{2}$ form a convex complete graph $K_{n-p+3}$. Also, join each of these $n-p$ vertices to every vertex in $K_{p}$. We then place all the disjoint cycles $F^{*}$ in $K_{n-p+3}$ in the same manner as was done in Remark 1 (with $v_{0}, v_{2}, v_{1}$ playing the roles of $x, y, z$ respectively) and obtain a triangulation $T^{*}$ for $K_{n-p+3}-F^{*}$ (since $v_{0}, v_{1}, v_{2}$ are isolated vertices in $\left.K_{n-p+3}-F^{*}\right)$. Then $T_{0} \cup T_{1} \cup T_{2} \cup T^{*}$ is a triangulation for $K_{n}-F_{n}$.

Now assume that $F^{*}$ is a 3 -cycle. Insert 3 vertices $u, v, w$ into the edges $v_{0} v_{1}, v_{1} v_{2}, v_{2} v_{3}$ respectively so that $\left\{v_{0}, u, v_{1}, v, v_{2}, w, v_{3}\right\}$ becomes the vertex set of a convex complete graph $K_{7}$. Also, join $u, v, w$ each to every vertex of $K_{p}$. Take $u v w u$ to be the 3 -cycle $F^{*}$. Note that the subgraph induced by $\left\{v_{0}, u, v_{1}, v, v_{2}, w, v_{3}, \ldots, u_{\beta}\right\}$ (recall that $\beta=\lceil n / 3\rceil$ ) is of the form $K_{\beta+3}-F^{*}$ which admits a triangulation $T_{0}^{*}$ (since $v_{0}, v_{1}, v_{2}, \ldots, v_{\beta}$ are isolated vertices in $\left.K_{\beta+3}-F^{*}\right)$. As such $T_{0}^{*} \cup T_{1} \cup T_{2}$ is a triangulation for $K_{n}-F_{n}$.

Case (2): $p=7$.

Let $C$ be the 7-cycle $v_{0} v_{3} v_{1} v_{6} v_{4} v_{2} v_{5} v_{0}$. Let $T_{p}$ be the triangulation of $K_{7}-F_{7}$ with 4 diagonals $v_{0} v_{2}, v_{2} v_{6}, v_{6} v_{3}, v_{3} v_{5}$. Adopt the same method of construction as was done in Case (1)(i) to obtain a triangulation $T_{p} \cup T^{*}$ for $K_{n}-F_{n}$ if $\left|V\left(F^{*}\right)\right| \geq 4$ and a triangulation $T_{p} \cup\left\{v_{0} u v_{1} v v_{2} w v_{3}\right\}$ for $K_{n}-F_{n}$ if $F^{*}$ is a 3 -cycle.

Case (3): $p=6$.

Let $C$ be the 6-cycle $v_{0} v_{2} v_{5} v_{3} v_{1} v_{4} v_{0}$.

Assume first that $\left|V\left(F^{*}\right)\right| \geq 4$. Insert $n-p$ vertices into the edges $v_{0} v_{1}, v_{0} v_{5}$ so that these $n-p$ vertices together with $v_{0}, v_{1}, v_{5}$ form a convex complete graph $K_{n-3}$. We then place all the disjoint cycles $F^{*}$ in this $K_{n-3}$ in the same manner as was done in 
Remark 1 (with $v_{5}, v_{1}, v_{0}$ playing the roles of $x, y, z$ respectively). Also, join the vertices of $F^{*}$ to every vertex in $v_{0}, v_{1}, \ldots, v_{5}$ to obtain the convex graph $K_{n}-F_{n}$. Let $V\left(F_{1}^{*}\right)$ and $V\left(F_{2}^{*}\right)$ denote the sets of vertices of $F^{*}$ which are placed on the edges $v_{0} v_{1}$ and $v_{0} v_{5}$ respectively. Consider the subgraphs $G_{0}$ and $G_{1}$ induced by $V\left(F_{1}^{*}\right) \cup\left\{v_{0}, v_{1}, v_{2}, v_{3}\right\}$ and $V\left(F_{2}^{*}\right) \cup\left\{v_{0}, v_{5}, v_{4}, v_{3}\right\}$ respectively. By Theorem 1, $G_{i}$ admits a triangulation $T_{i}$, $i=0,1$. Consequently, $T_{0} \cup T_{1} \cup\left\{v_{0} v_{3}\right\}$ is a triangulation for $K_{n}-F_{n}$.

Now suppose $F^{*}$ is a 3 -cycle. Here we take $F_{n}=C_{6} \cup C_{3}$ where $C_{6}=v_{0} v_{3} v_{8} v_{5} v_{2} v_{6} v_{0}$ and $C_{3}=v_{1} v_{4} v_{7} v_{1}$. Then a triangulation for $K_{n}-F_{n}$ is given by the set of diagonals $\left\{v_{5} v_{0}, v_{0} v_{7}, v_{7} v_{5}, v_{5} v_{1}, v_{1} v_{3}, v_{3} v_{5}\right\}$.

Case (4): $p=5$.

Let $C$ be the 5 -cycle $v_{0} v_{2} v_{4} v_{1} v_{3} v_{0}$.

Assume first that $\left|V\left(F^{*}\right)\right| \geq 4$. Insert $n-5$ vertices into the edges $v_{0} v_{1}, v_{1} v_{2}$ so that these $n-5$ vertices together with $v_{0}, v_{1}, v_{2}$ form a convex complete graph $K_{n-2}$. We then place all the disjoint cycles $F^{*}$ in this $K_{n-2}$ in the same manner as was done in Remark 1 (with $v_{0}, v_{2}, v_{1}$ playing the roles of $x, y, z$ respectively). Also, join all these $n-5$ vertices to each of the vertex in $\left\{v_{0}, v_{1}, \ldots, v_{4}\right\}$ so that the resulting convex graph is $K_{n}-F_{n}$. Let $v_{0} w$ be the boundary edge of $K_{n-2}$ where $w \in V\left(F^{*}\right)$. Let $G^{*}$ denote the subgraph of $K_{n}-F_{n}$ induced by the vertices $V\left(F^{*}\right) \cup\left\{v_{1}, v_{2}, v_{3}\right\}$. Since $v_{2}$ is an isolated vertex in $G^{*}, G^{*}$ admits a triangulation $T^{*}$. As such $T^{*} \cup\left\{w v_{0}, v_{0} v_{4}, v_{4} w, v_{3} v_{4}\right\}$ is a triangulation of $K_{n}-F_{n}$.

Hence assume that $F^{*}$ is a 3 -cycle. Here we take $F_{n}=C_{5} \cup C_{3}$ where $C_{5}=$ $v_{0} v_{3} v_{6} v_{2} v_{5} v_{0}$ and $C_{3}=v_{1} v_{4} v_{7} v_{1}$. Then a triangulation for $K_{n}-F_{n}$ is given by the set of diagonals $\left\{v_{0} v_{2}, v_{2} v_{4}, v_{4} v_{0}, v_{0} v_{6}, v_{4} v_{6}\right\}$.

Case (5): $p=4$.

In view of the preceding cases, we may assume that $F_{n}$ is a disjoint union of cycles each of length at most 4 .

Suppose $F_{n}=C_{4} \cup C_{4}$. Then we may take $F_{n}$ to be the two disjoint 4-cycles given by $v_{0} v_{2} v_{5} v_{3} v_{0}$ and $v_{1} v_{6} v_{4} v_{7} v_{1}$. Then a triangulation for $K_{8}-F_{8}$ is given by the set of diagonals $v_{1} v_{3}, v_{1} v_{4}, v_{1} v_{5}, v_{5} v_{0}, v_{5} v_{7}$.

Hence we assume that $\left|V\left(F^{*}\right)\right| \geq 6$. Take a convex complete graph $K_{n-1}$ and place all the disjoint cycles $F^{*}$ in $K_{n-1}$ in the same manner as was done in Remark 1, Now insert a new vertex $w$ on a boundary edge incident to $z$ and join $w$ to all vertices of $K_{n-1}$ to obtain a convex complete graph $K_{n}$. Let $C$ denote the cycle $x z y w x$. Let $V\left(F_{1}^{*}\right)$ denote the set of vertices in $F^{*}$ which are placed on the boundary edge $x z$, and let $V\left(F_{2}^{*}\right)$ denote the set of vertices in $F^{*}$ which are placed on the boundary edge $w y$.

Let $x_{1} \in V\left(F_{1}^{*}\right)$ and $x_{2} \in V\left(F_{2}^{*}\right)$ such that $x_{1} x_{2}$ is not an edge in $F^{*}$. Partition $K_{n}-F_{n}$ into three convex graphs $G_{1}, G_{2}$ and $G_{3}$ induced by the vertices $\left\{x_{1}, \ldots, z\right\}$, $\left\{z, \ldots, x_{2}\right\}$ and $\left\{x_{2}, \ldots, y, x, \ldots, x_{1}\right\}$. Then it is easy to see that $z$ is an isolated in $G_{1}$ and $G_{2}$ and each $x$ and $y$ is an isolated vertex in $G_{3}$. Let $T_{i}$ denote a triangulation of 
$G_{i}, i=1,2,3$. Then a triangulation for $K_{n}-F_{n}$ is given by $T_{1} \cup T_{2} \cup T_{3}$.

Case (6): $p=3$.

In view of the preceding results, we just need to consider the case $F_{n}=C_{3} \cup C_{3} \cup$ $\cdots \cup C_{3}$ and $n \geq 9$.

Let $\alpha=n / 3$ and take $F_{n}=\left\{v_{i} v_{i+\alpha} v_{i+2 \alpha} v_{i} \mid i=0,1, \ldots, \alpha-1\right\}$.

For $i=0,1,2$, let $G_{i}$ denote the convex complete subgraph induced by the vertices $v_{i(\alpha-1)}, v_{i(\alpha-1)+1}, \ldots, v_{(i+1)(\alpha-1)}$. Also, let $G_{3}$ denote the convex complete subgraph induced by the vertices $v_{3(\alpha-1)}, v_{3 \alpha-2}, \ldots, v_{0}$.

Each $G_{i}, i=0,1,2,3$ admits a triangulation $T_{i}$ if $n \geq 12$. Hence $T_{0} \cup T_{1} \cup T_{2} \cup T_{3} \cup$ $\left\{v_{0} v_{\alpha-1} v_{2(\alpha-1)} v_{3(\alpha-1)} v_{0}, v_{0} v_{2(\alpha-1)}\right\}$ yields a triangulation for $K_{n}-F_{n}$ if $n \geq 12$. If $n=9$, a triangulation for $K_{9}-F_{9}$ is given by the diagonals $\left\{v_{0} v_{2}, v_{2} v_{4}, v_{4} v_{0}, v_{4} v_{6}, v_{6} v_{8}, v_{8} v_{4}\right\}$.

This completes the proof.

\section{$5 \quad$ Regular graphs}

In view of the results in the preceding section, it is natural to ask which regular graph is potentially triangulable in $K_{n}$.

Problem 1 Let $r \geq 3$ be a natural number and let $G$ be an $r$-regular graph with $n$ vertices. It is true that there is a natural number $n_{0}(r)$ such that when $n \geq n_{0}(r)$, then $G$ is potentially triangulable in the convex complete graph $K_{n}$ ?

We believe that the above problem is true. However we do not have a complete answer for this even when restricted to the case $r=3$. Nevertheless we offer the following special case of a 3-regular graph which is well-known in the literature.

Suppose $n$ and $k$ are two integers such that $1 \leq k \leq n-1$ and $n \geq 5$. The generalized Petersen graph $P(n, k)$ is defined to have vertex-set $\left\{a_{i}, b_{i}: i=0,1, \ldots, n-1\right\}$ and edgeset $E_{1} \cup E_{2} \cup E_{3}$ where $E_{1}=\left\{a_{i} a_{i+1}: i=0,1, \ldots, n-1\right\}, E_{2}=\left\{b_{i} b_{i+k}: i=0,1, \ldots, n-1\right\}$ and $E_{3}=\left\{a_{i} b_{i}: i=0,1, \ldots, n-1\right\}$ with subscripts reduced modulo $n$. Edges in $E_{3}$ are called the spokes of $P(n, k)$.

Proposition 3 Suppose $1 \leq k<n / 2$. Then the generalized Petersen graph $P(n, k)$ is potentially triangulable in the convex complete graph $K_{2 n}$ where $n \geq 5$.

Proof: Let the vertices of $K_{2 n}$ be denoted $v_{1}, v_{2}, v_{3}, \ldots, v_{2 n}$. We shall pack $P(n, k)$ on $K_{2 n}$ so that $K_{2 n}-P(n, k)$ admits a triangulation.

Case (1): $k=1$

In this case, $P(n, 1)$ consists of two $n$-cycles $C=a_{0} a_{1} a_{2} \cdots c_{n-1} a_{0}$ and $C^{\prime}=b_{0} b_{1} b_{2} \cdots$ $b_{n-1} b_{0}$ together with the edges $a_{i} b_{i}, i=0,1,2, \ldots, n-1$. 
If $n$ is even, we place $C$ on $K_{2 n}$ so that $C$ takes the form

$$
v_{1} v_{3 n / 2} v_{2} v_{(3 n-2) / 2} v_{3} v_{(3 n-4) / 2} \cdots v_{(n-2) / 2} v_{n+2} v_{n / 2} v_{n+1} v_{1}
$$

and that $C^{\prime}$ takes the form

$$
v_{(3 n+2) / 2} v_{n} v_{(3 n+4) / 2} v_{n-1} v_{(3 n+6) / 2} v_{n-2} \cdots v_{(n+4) / 2} v_{2 n} v_{(n+2) / 2} v_{(3 n+2) / 2}
$$

with spokes given by $v_{(3 n+2 i) / 2} v_{i}, i=1,2, \ldots, n / 2$ and $v_{(3 n-2 i) / 2} v_{n-i}, i=0,1,2, \ldots, n / 2-$ 1.

If $n$ is odd, we place $C$ on $K_{2 n}$ so that $C$ takes the form

$$
v_{1} v_{(3 n-1) / 2} v_{2} v_{(3 n-3) / 2} v_{3} v_{(3 n-5) / 2} \cdots v_{n+2} v_{(n-1) / 2} v_{n+1} v_{(n+1) / 2} v_{1}
$$

and that $C^{\prime}$ takes the form

$$
v_{(3 n+1) / 2} v_{n} v_{(3 n+3) / 2} v_{n-1} v_{(3 n+5) / 2} v_{n-2} \cdots v_{2 n-1} v_{(n+3) / 2} v_{2 n} v_{(3 n+1) / 2}
$$

with spokes given by $v_{(3 n+2 i-1) / 2} v_{i}, \quad i=1,2, \ldots,(n+1) / 2$, and $v_{(3 n-2 i-1) / 2} v_{n-i}$, $i=0,1,2, \ldots,(n-3) / 2$.

In both cases consider the subgraph graphs $G_{1}$ and $G_{2}$ induced by the sets of vertices $\left\{v_{1}, v_{2}, \ldots, v_{n}\right\}$ and $\left\{v_{n+1}, v_{n+2}, \ldots, v_{2 n-1}, v_{2 n}\right\}$ respectively. Since the vertex $v_{2}$ in $G_{1}$ (likewise the vertex $v_{n+1}$ in $G_{2}$ ) is not incident with any edges from $G_{1}$ (respectively $G_{2}$ ) since $n \geq 5, G_{i}$ admits a triangulation $T_{i}, i=1,2$. A triangulation of $K_{2 n}-P(n, 1)$ is given by $T_{1} \cup T_{2} \cup\left\{v_{n} v_{2 n}, v_{1} v_{2 n}, v_{n} v_{n+1}\right\}$.

Case (2): $1<k<n / 2$

Place $E_{1}$ and $E_{2}$ on $K_{2 n}$ so that $E_{1}$ takes the form $v_{2} v_{4} v_{6} \ldots v_{2 n-2} v_{2 n} v_{2}, E_{2}$ takes the form $\left\{v_{i} v_{i+2 k}: i=1,3, \ldots, 2 n-1\right\}$ and $E_{3}$ takes the form $\left\{v_{2 i} v_{2 i+3}: i=1,2, \ldots, n\right\}$. Here the operations on the subscripts are reduced modulo $2 n$.

Consider the subgraph $G$ induced by the vertices $\left\{v_{4}, v_{5}, v_{6}, \ldots, v_{2 n-3}\right\} \cup\left\{v_{0}, v_{1}, v_{2 n-1}\right\}$. Since the vertex $v_{0}$ is not adjacent to every vertex in $G, G$ admits a triangulation $T_{G}$. Then $T_{G} \cup\left\{v_{1} v_{2} v_{3} v_{4}\right\} \cup\left\{v_{1} v_{3}\right\} \cup\left\{v_{2 n-3} v_{2 n-2} v_{2 n-1}\right\}$ is a triangulation for $K_{2 n}-P(n, k)$.

This completes the proof.

\section{An application}

The skewness of a graph $G$, denoted $s k(G)$, is the minimum number of edges to be deleted from $G$ such the resulting graph is planar. In [6], Kainen proves that all graphs $G$ with $\operatorname{sk}(G) \leq 2$ are 5-colorable (and the result is best possible). Also, in the same paper he proves that if $s k(G) \leq 5$, then $G$ is 6 -colorable. In the following year, in [7, it was shown that if $s k(G)<\left(\begin{array}{l}k \\ 2\end{array}\right)$, then $G$ is $(2+k)$-colorable for $k \geq 3$. This result was generalized to other orientable surfaces in [9].

For more details about the notion of skewness of a graph and for some recent results, the reader may consult the papers [2, [3] and [4]. 
Definition 5 The geometric skewness of a geometric graph $G$, denoted $s k_{g}(G)$ is the minimum number of edges to be removed from $G$ so that the resulting graph can be redrawn as a geometric planar graph. The convex skewness of a convex graph $G$, denoted $s k_{c}(G)$ is the minimum number of edges to be removed from $G$ so that the resulting graph is a convex plane graph.

Theorem 6 For any geometric graph $G, s k_{g}(G)=s k(G)$.

Proof: The proof follows immediately from Fáry's theorem ([5] $)$ which states that any simple planar graph can be drawn on the plane without crossings so that its edges are straight line segments.

Proposition 4 Let $F$ be a subgraph of a convex complete graph $K_{n}$. Suppose $K_{n}-F$ admits a triangulation. Then $s k_{c}\left(K_{n}-F\right)=\left(\begin{array}{c}n-2 \\ 2\end{array}\right)-|E(F)|$.

Proof: It is known that the number of edges in a triangulation $T$ of a convex $n$-gon is $2 n-3$ (with $n-3$ of them being non-boundary edges). If any new straight line segment is added to the triangulation, it will intersect with some non-boundary edge of $T$. Hence, if $K_{n}-F$ admits a triangulation, then we have $s k_{c}\left(K_{n}-F\right)=\left|E\left(K_{n}\right)\right|-|E(F)|-(2 n-3)$ which yields $s k_{c}\left(K_{n}-F\right)=\left(\begin{array}{c}n-2 \\ 2\end{array}\right)-|E(F)|$.

\section{References}

[1] A. Aichholzer, S. Cabella, R. Fabila-Monroy, D. Flores-Peñaloza, T. Hackl, C. Huemer, F. Hurtado and D.R. Wood, Edge-removal and noncrossing configurations and geometric graph, Discrete Maths. \& Theorectical Comput. Sci., 12 (2010) 75-86.

[2] G.L. Chia and C.L. Lee, Skewness and crossing numbers of graphs, Bull. Inst. Combin. Appl. Vol. 55 (2009) 17-32.

[3] G.L. Chia and K.A. Sim, On the skewness of the join of two graphs, Discrete Appl. Math. Vol. 161. (2013) 2405 - 2409.

[4] R.J. Cimikowski, Graph planarization and skewness, Congr. Numer. 88 (1992) 2132 .

[5] I. FÁRY, On straight-line representation of planar graphs, Acta Sci. Math. (Szeged), 11 (1948) 229 - 233.

[6] P.C. Kainen, A generalization of the 5-color theorem, Proc. Amer. Math. Soc., 45 (1974) 450-453. 
[7] P.C. Kainen, Chromatic number and skewness, J. Combinat. Theory, 18 (1975) $32-34$.

[8] E.L. Lloyd, On triangulations on a set of points in the plane, Proc. of IEEE Symposium on Foundations of Computer Science (FOCS), IEEE (1977) 228-240.

[9] H. Pahlings, On the chromatic number of skew graphs, J. Combinat. Theory, 25 (1978) 303-306. 